\title{
Modelling texturised cast structures in the forging of superalloys
}

\author{
Jan Terhaar ${ }^{1, a}$, Nikolaus Blaes ${ }^{1}$, Dieter Bokelmann ${ }^{1}$ and Hendrik Schafstall ${ }^{2, b}$ \\ ${ }^{1}$ Saarschmiede GmbH Freiformschmiede, Bismarckstraße 57-59, 66333 Völklingen, Germany \\ ${ }^{2}$ simufact engineering $\mathrm{GmbH}$, Tempowerkring 3, 21079 Hamburg, Germany \\ aj.terhaar@saarschmiede.com, ${ }^{b}$ hendrik.schafstall@simufact.de
}

Key words: vacuum arc remelting, solidification texture, anisotropy, forging, simulation, alloy 718

\begin{abstract}
The main objective of remelting processes commonly used in the production of superalloys is to obtain a columnar dendritic solidification structure throughout the whole ingot. Besides reduced microsegregation, this cast structure features a preferred orientation, which is depending on the primary dendrites' growth direction and therefore closely related to the ingot's pool shape. As a result, non-isotropic material behaviour can be observed during initial forging operations. Since the correct prediction of material flow is a prerequisite for the further analysis of forging processes by means of numerical simulation, the solidification texture's influence on plastic flow was accounted for by the application of an anisotropic material model. The model according to Barlat was used to scale the flow stress with respect to the crystal orientations observed in the examination of vacuum arc remelted alloy 718, thereby considering the flow stress' dependency on strain, strain rate and temperature. The parameters defining the material's anisotropy could be determined by the upsetting of cylindrical specimen from a remelted ingot.
\end{abstract}

\section{Introduction}

The possibilities to optimise bulk metal forming processes with the help of process simulation are limited by the fact that the material commonly is modelled as a homogeneous, isotropic continuum. In terms of primary forging operations, this assumption introduces a certain fuzziness in the calculation results, since the material's yield behaviour is strongly affected by the inhomogenities of a cast structure. In superalloys - or remelted material in general - another important aspect has to be considered. Due to the columnar dendritic structure, which ideally is maintained throughout the whole cross-section of an ingot, the remelting process promotes the development of a texture, leading to anisotropic properties in terms of plastic flow. The distribution of the primary dendrites' axes, which is determined by the pool shape, is controlled by the remelting conditions. Thus, it is obvious that the solidification conditions not only have an effect on the materials segregation but also on its behaviour during plastic deformation. It is likely that the existing solidification texture, if it was accounted for in the numerical simulation, would lead to a different stress-strain state in the material, as compared to the isotropic case. Concerning superalloys, this issue is of particular importance since these alloys react comparably sensitive on process conditions. The intention of this work is to present an approach, how this issue can be addressed in the modelling of hot working processes, where the flow stress has to be considered as a function of strain, strain rate and temperature.

Background. The mentioned anisotropic flow behaviour has already been observed and commented on by other authors [1,2], when plastic properties of remelted superalloys were examined by using material in the as-cast or homogenized state, i.e. when the samples have not been subjected to prior deformation. In cylindrical specimens for upsetting tests, this expresses in elliptical barrelling. Nevertheless, it was not attempted to find a connection between solidification conditions and this feature of the materials' plastic yield that actually can be used in the numerical simulation of primary forging operations. There are several possible explanations to the fact, that the elliptical shape of upset specimens could not yet directly be linked to the solidification texture of a remelted ingot. On the one hand, to reveal a possible correlation, the specimens' orientation and position in the ingot have to be marked prior to sampling. On the other hand, the size of the specimens has to 
cover a sufficient number of grains. However, if the material has undergone a diffusion annealing, which is a standard treatment for superalloys, significant grain growth in the material can lead to a situation where the sample dimensions usually used for upsetting tests only hold a very small number of grains. In that case, the elliptical shape of the specimen after upsetting can easily be interpreted as randomly oriented [1].

\section{Experimental Procedure}

In the experimental part of this investigation, several cylindrical specimens were taken from drill cores in the axial direction of a longitudinal segment of a $\varnothing 500 \mathrm{~mm}$ VAR ingot in alloy 718 . The drillings were evenly distributed on the outer part of the ingot's cross-section and one drill sample was taken from the centre. To avoid the mentioned problems, the specimens were sized $\varnothing 55.6 \times$ $100 \mathrm{~mm}$, so that forming could not be carried out on a normal testing machine. Instead, the upsetting of these large-sized specimens was done on an industrial 10MN hydraulic forging press. Before taking the samples, the radial direction was marked by two indentations on the front face of the drill cores as it is shown in Fig. 1. From each drill core two cylindrical specimens were taken and the marked radial direction was transferred to each of them. Since a possible influence of different homogenisation treatments should also be analysed, one core sample from the rim was left in the as-cast state while the others were annealed prior to machining the specimens for upsetting.

Upsetting Tests. The two specimens from each of the drill cores were then used to realise two different upset strains; 20\% and 50\% height reduction (upsetting to $80 \mathrm{~mm}$ and $50 \mathrm{~mm}$, resp.). The samples were heated to $1075^{\circ} \mathrm{C}$ in an electric furnace and equalised for $\sim 1.5 \mathrm{~h}$. After upsetting they were quenched in water immediately.
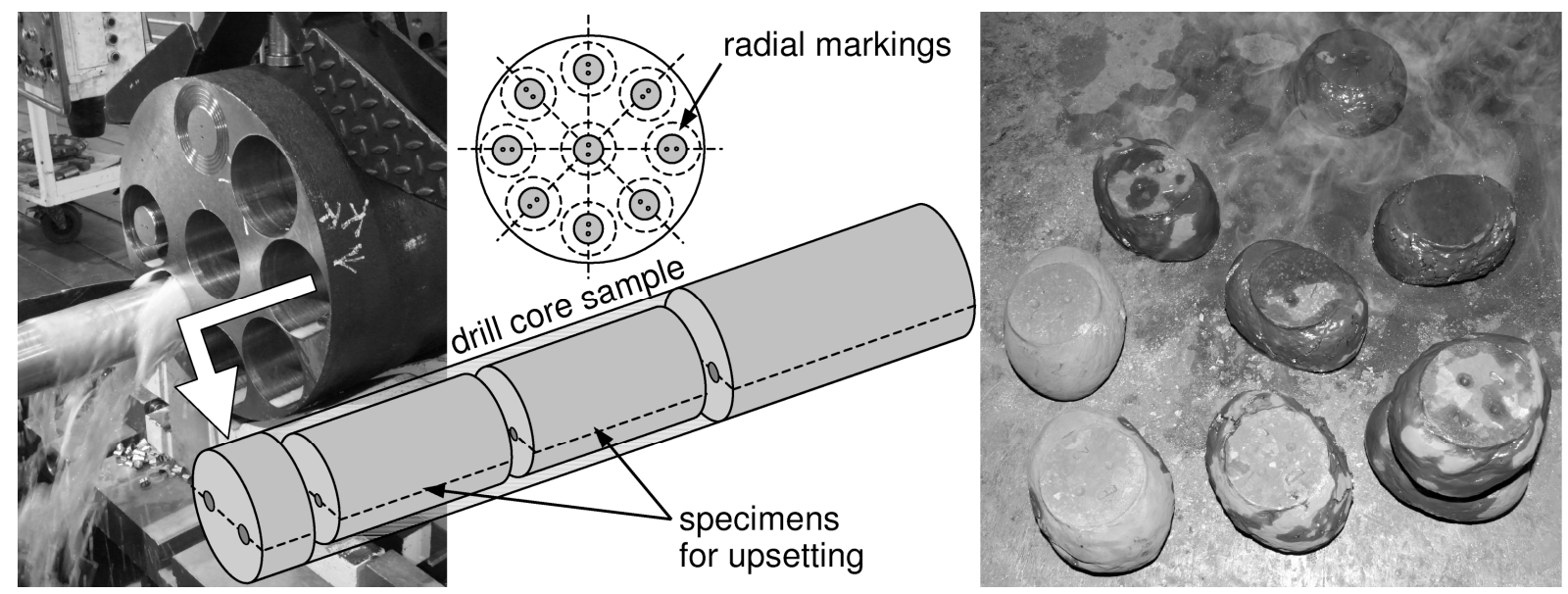

Fig. 1: Sampling (left) and specimens after upsetting with 50\% height reduction (right).

The upset rim samples clearly revealed that the elliptical barrelling had a preferred orientation; regardless of the different homogenisation treatments, in all specimens the minor diameter of the elliptical shape was aligned to the radial markings on the front face, indicating that the plastic flow preferentially follows the circumferential direction (= major diameter). This could be observed in both height reductions.

Microstructural Analysis. In another VAR trial batch the as-cast microstructure was analysed by means of electron backscatter diffraction (EBSD). It is well known, that face-centred cubic (fcc) materials like alloy 718 solidify along the crystallographic [100] direction. However, the analysed mid-radius position revealed that besides this [100] fibre texture the (111) slip planes also have a preferred orientation. By rotating the three-dimensional EBSD results towards the solidification direction, i.e. looking from the dendrites' root towards the melt pool, it became obvious that the body diagonals of the unit cell, which equal the normal direction to the slip planes, would mainly lie in the planes spanned by the vector of the primary dendrites' axes and the vector following the pool shape in radial, respectively peripheral direction. 


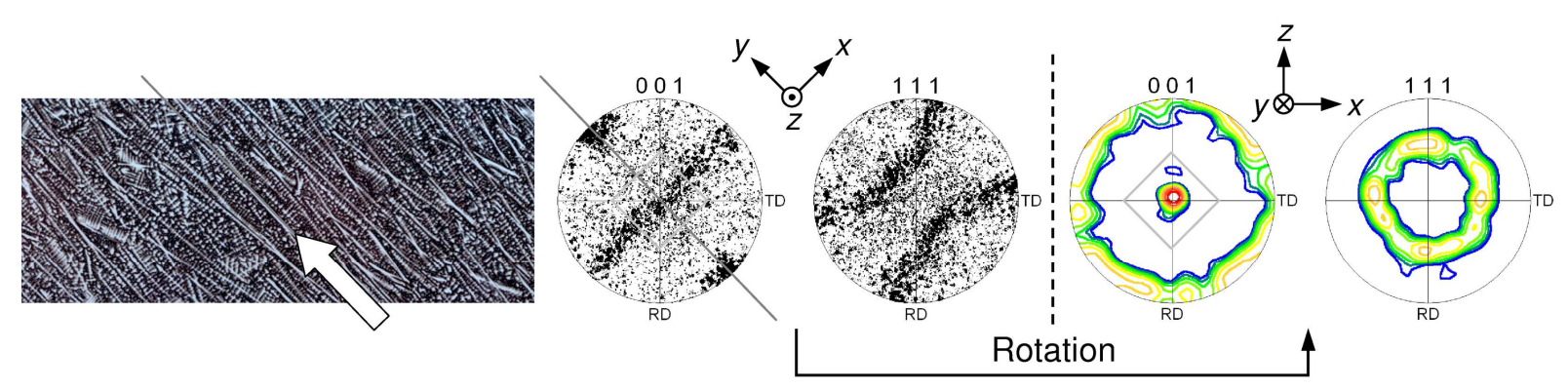

Fig. 2: Results of the metallographic and crystallographic analysis of the as-cast microstructure.

The microstructure is shown in Fig. 2 along with the corresponding discrete pole figures and the orientation distribution functions for the rotated data.

\section{Numerical Procedure}

Since obviously the distribution of solidification angles resulting from the pool shape during remelting has an influence on the material's yield behaviour, it was attempted to close the gap between these consecutive processes by transferring the results of the vacuum arc remelting (VAR) simulation into the finite element modelling of the specimen's upsetting.

Simulation of Vacuum Arc Remelting. The VAR process of the trial batch, from which the upsetting cylinders were taken, was simulated with the commercial software MeltFlow-VAR, which is capable of capturing transient effects introduced by the specified process conditions [3]. The entire process was calculated based on the real process data in order to gain information about the pool shape as a function of process duration. For a given solid fraction (here: 0.9), the isotherm defining the pool can be evaluated in terms of its slope, which can vary with time depending on the melt profile. Consequently, the solidification angle is available as field data for the whole ingot.

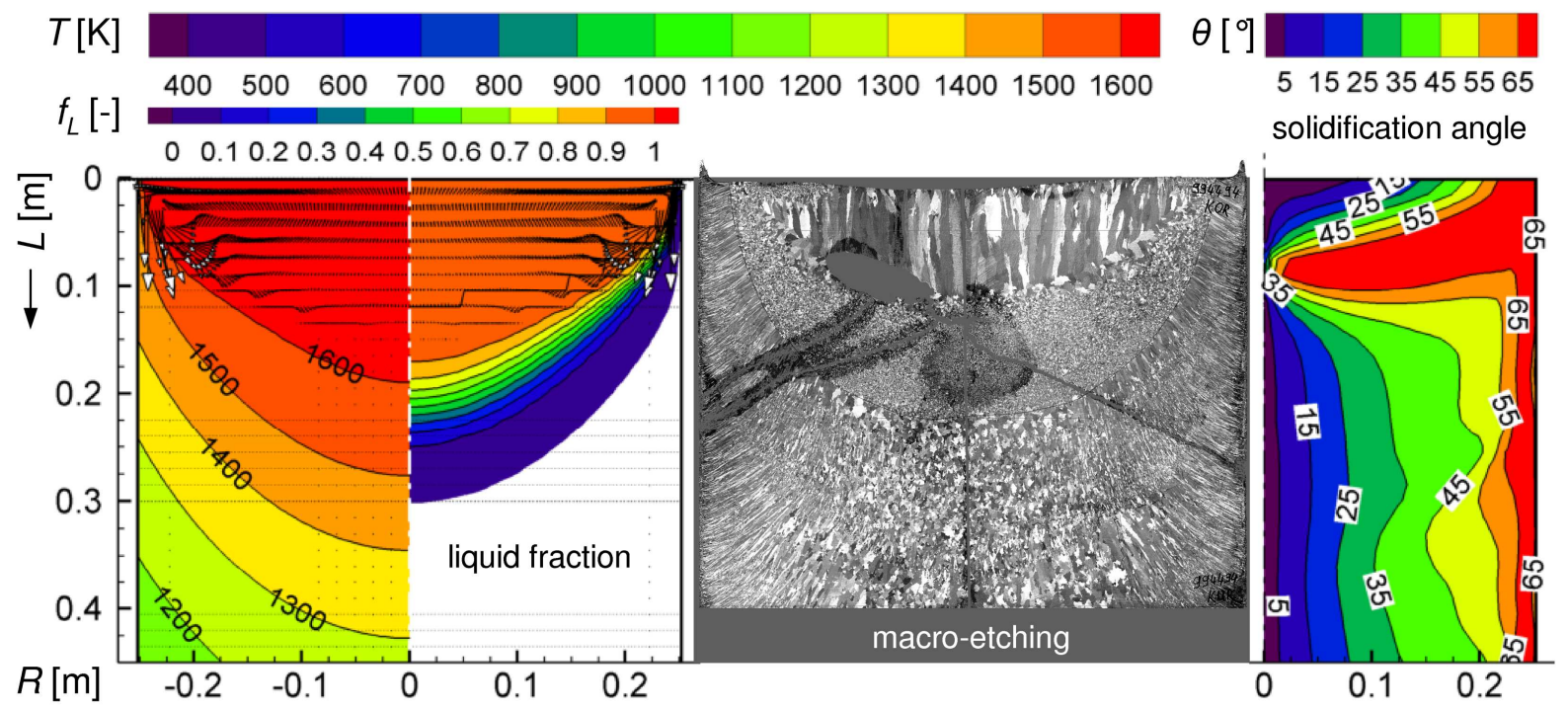

Fig. 3: Simulation results for the VAR batch from which the samples were taken.

The results of the simulation are presented in Fig. 3 for the top of the ingot. The pool shape at meltend is opposed to the corresponding macro-etched slices of the longitudinal section. Furthermore, the equivalent distribution of solidification angles is also shown.

Modelling the Solidification Structure. The geometrical data of the iso-contour lines for the calculated orientation of the primary dendrites was exported, processed and read in by the software used for the forging simulation, simufact.formingSFM. The curves were then revolved to create isosurfaces of the discretised angles in the pre-processor of the simulation tool. By positioning the finite element model of the upsetting specimen at the 'exact' position, where the drill cores were taken, the surfaces could be used to assign every element of the model a certain angle (see Fig. 4). 


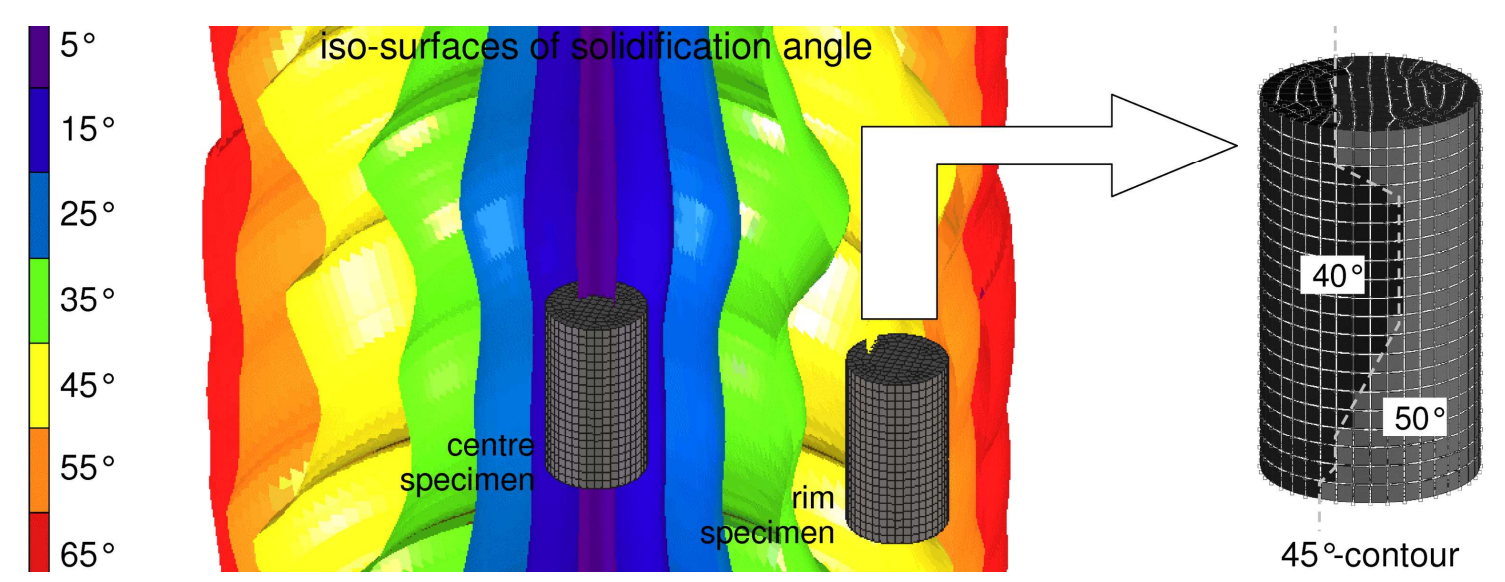

Fig. 4: Transfer of solidification angles to the finite element model for the upsetting simulation.

When using anisotropic properties in the modelling of forming processes, each finite element possesses its own coordinate system defining the axes of anisotropy. Since anisotropic material laws commonly find their application in cold forming processes of sheet metal, the elements' coordinate axes represent planar anisotropy. In order to model the cast structure, different orientations of these coordinate systems had been tested, where the rotations for the 2880 hexahedral elements in the model were performed automatically by a Python script. Finally, the best results were achieved by rotating the axes of anisotropy according to Fig. 5.
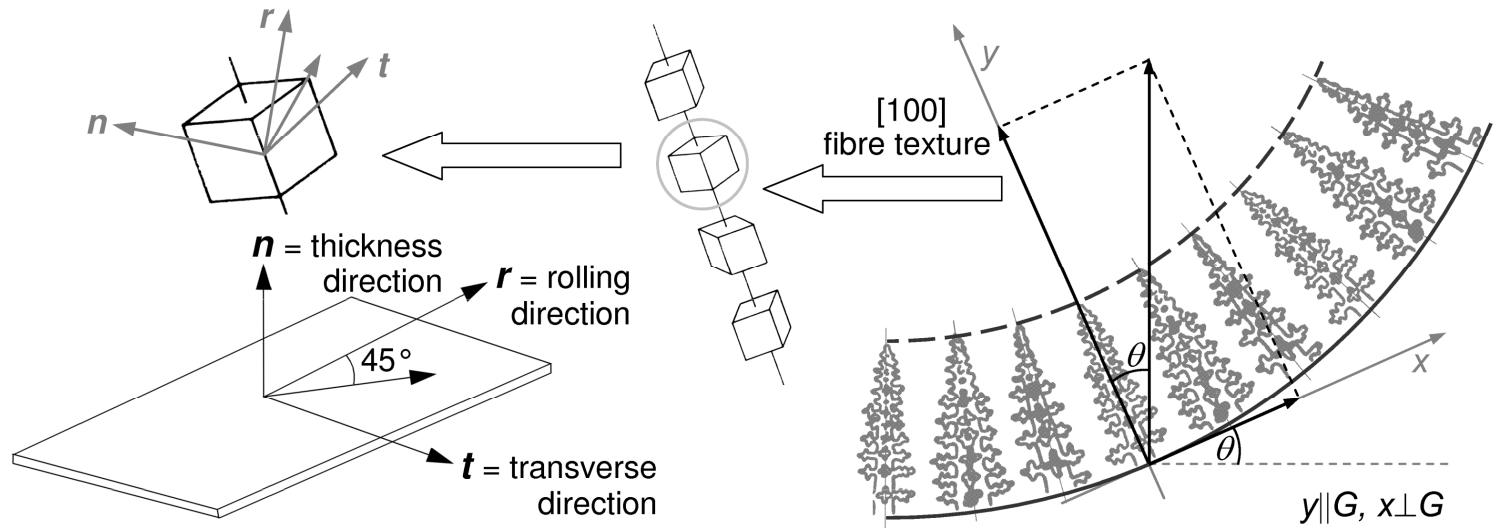

Fig. 5: Connection between solidification angles and orientation of the element coordinate system.

The yield criterion used to model the orientation dependency in three-dimensional deformation is the one defined by Barlat et al. given as [4]

$$
f=\left|s_{1}-s_{2}\right|^{m}+\left|s_{2}-s_{3}\right|^{m}+\left|s_{3}-s_{1}\right|^{m}=2 \sigma^{m}
$$

where $s_{i=1,2,3}$ are the principal values of the symmetric matrix $S_{i, j}$ defined as

$$
S=\left[\begin{array}{ccc}
\frac{c_{3}\left(\sigma_{x x}-\sigma_{y y}\right)-c_{2}\left(\sigma_{z z}-\sigma_{x x}\right)}{3} & c_{6} \sigma_{x y} & c_{5} \sigma_{z x} \\
c_{6} \sigma_{x y} & \frac{c_{1}\left(\sigma_{y y}-\sigma_{z z}\right)-c_{3}\left(\sigma_{x x}-\sigma_{y y}\right)}{3} & c_{4} \sigma_{z y} \\
c_{5} \sigma_{z x} & c_{4} \sigma_{z y} & \frac{c_{2}\left(\sigma_{z z}-\sigma_{x x}\right)-c_{1}\left(\sigma_{y y}-\sigma_{z z}\right)}{3}
\end{array}\right] .
$$

The symmetry axes $(x, y, z)$ represent the orthogonal axes of anisotropy, which are aligned with the initial rolling, transverse and normal direction (see Fig. 5). The material coefficients $c_{i=1-6}$ in Eq. 2 constitute the anisotropic properties. For $c_{i=1-6}=1$ the material becomes isotropic and Eq. 1 reduces to the von Mises yield criterion with $m=2$ (or 4). Increasing the value of $m$ results in a decrease of the curvature of the rounded vertices near the uniaxial and balanced tension ranges of the yield 
surface. The exponent $m$ that is mainly associated with the material's crystal structure is recommended to equal 8 for fcc materials. With the assumption of $c_{4}=c_{5}=1$ (isotropic properties for transverse directions), there are four unknown independent coefficients $c_{1}, c_{2}, c_{3}$ and $c_{6}$. If experimental data is available, these coefficients can be calculated from $\sigma_{0}, \sigma_{45}, \sigma_{90}$ and $\sigma_{b}$, representing the tensile yield stresses at $0^{\circ}, 45^{\circ}$ and $90^{\circ}$ from the rolling direction and the balanced biaxial yield stress, respectively [5]. Since in bulk metal forming the flow stress generally is measured in compression tests, this data was not readily available. However, due to the fact that simufact engineering created the possibility to use this yield criterion under hot forming conditions, the flow stress could be scaled in the different directions while maintaining the dependency of strain, strain rate and temperature. Hence, existing flow stress data determined from compression tests under usual hot forming conditions $\left(\varphi \leq 1.0, d \varphi / d t=0.01 \ldots 1.0 \mathrm{~s}^{-1}, 875 \ldots 1125^{\circ} \mathrm{C}\right)$ could be used. With the described orientation of the elements' coordinate system, only the $\sigma_{45} / \sigma_{0}$ ratio had to be altered to obtain the elliptical barrelling as it was observed experimentally. Since this $\sigma_{45}$-direction equals the normal vector to the octahedral planes, it is rather unfavourably oriented to activate the main slip planes. Hence, the flow stress in this direction had to be raised slightly in contrast to $\sigma_{0}$ and $\sigma_{90}$, which is consistent with the results of the microstructural analysis.
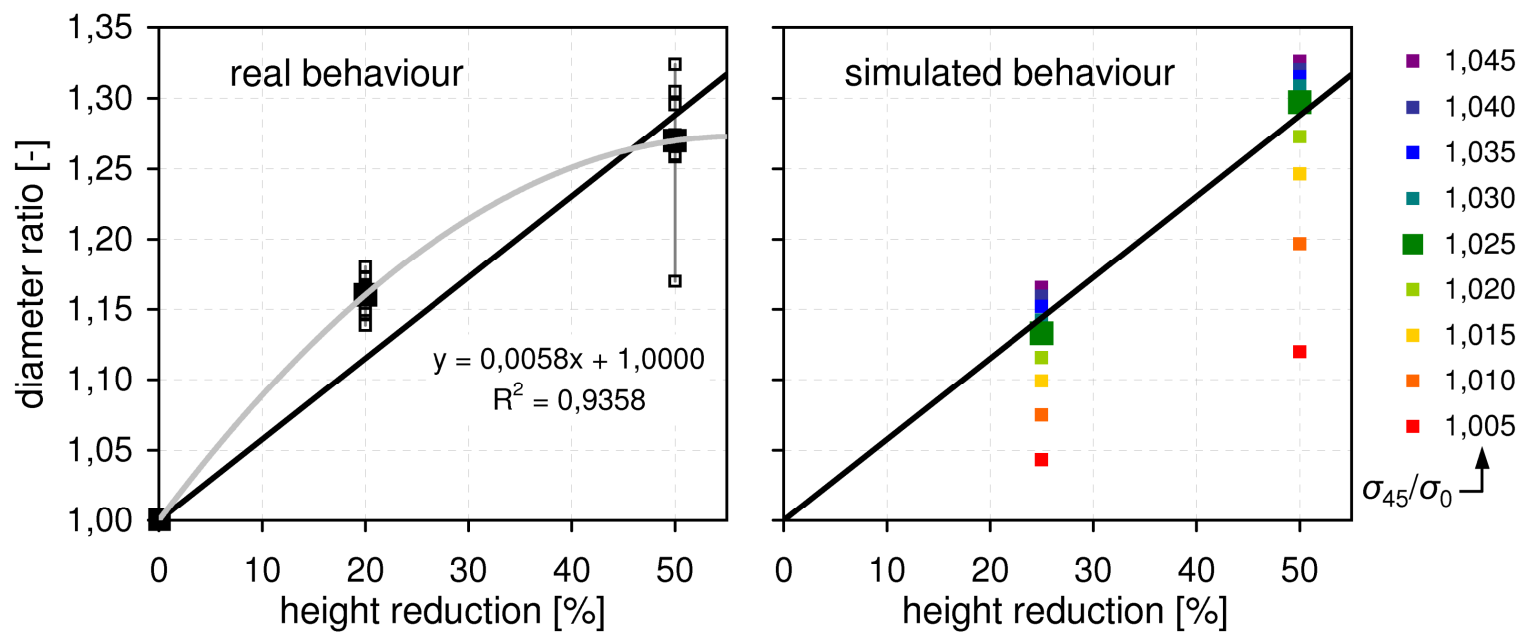

Fig. 6: Calibration of the anisotropic yield criterion based on measured diameter ratios.

To obtain the best fit with the experimental results, a series of thermo-mechanically coupled simulations of the upsetting was carried out while altering the $\sigma_{45} / \sigma_{0}$ ratio. From the results of these calculations the diameter ratio $d_{\text {major }} / d_{\text {minor }}$ was determined and compared with the experimental values. Best agreement concerning the dimensions of the barrelling ellipse could be achieved with scaling up the flow stress $\sigma_{45}$ by $2.5 \%$ (see Fig. 6), which equals the following set of coefficients in the yield criterion: $m=8 ; \sigma_{45} / \sigma_{0}=1.025, \sigma_{90} / \sigma_{0}=\sigma_{b} / \sigma_{0}=1.0 \rightarrow c_{i=1-5}=1.0$ and $c_{6}=0.967157$.

Given the usual scattering in the flow stress of as-cast or coarse-grained homogenized material, this scaling is almost negligible. However, it has to be pointed out, that the anisotropic deformation behaviour could only be reproduced in the simulation by using a kinematic work hardening approach in the interpretation of the given flow stress data.

\section{Results and Discussion}

The quite realistic representation of the upset specimen's shape (rim position) is shown in Fig. 7.
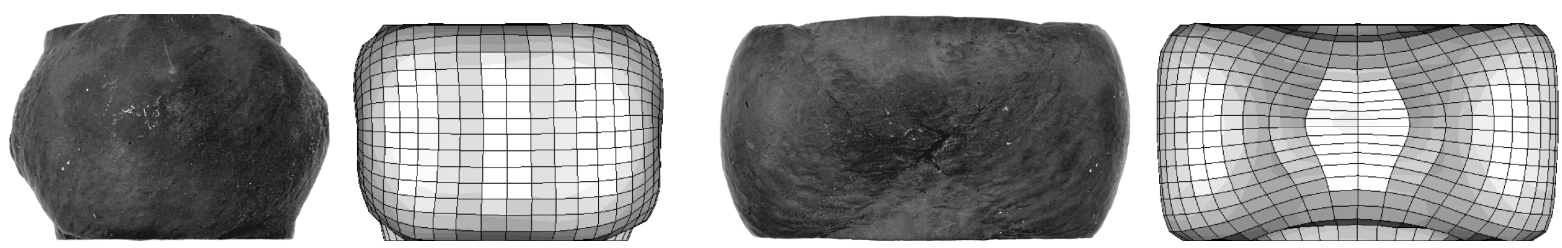

Fig. 7: Comparison of real (as-cast) and simulated material behaviour; $d_{\text {minor }}$ (left) and $d_{\text {major }}$ (right). 
It is obvious that recrystallisation, which this simulation does not account for, also affects the deformation behaviour. Most likely that explains the different shape of the specimen's outline along the minor diameter. An overview on the effect of the different material models is given in Fig. 8. The comparison between real (homogenized state) and simulated deformation shows that the anisotropic yield criterion leads to some deviation in the centre position. This is due to the fact, that there was a significant amount of equiaxed solidification in the central region of the used trial batch.

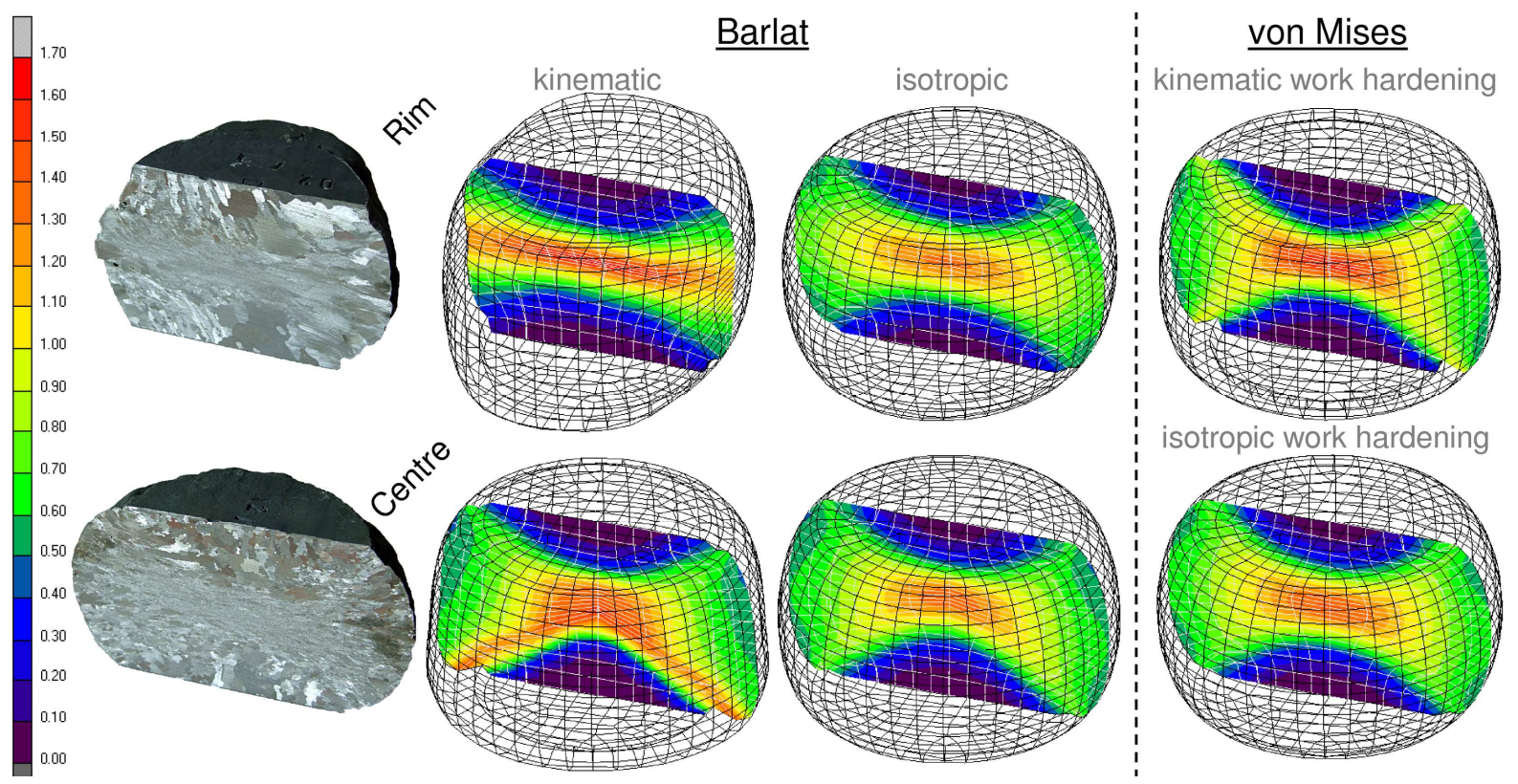

Fig. 8: Comparison of the strain distributions calculated with different yield criteria.

In that case, congruence can be improved by changing the treatment of work hardening behaviour from 'kinematic' to 'isotropic'. Of course, in this rather isotropic centre specimen, the application of the common von Mises approach shows good results, too.

\section{Summary}

It was shown, that there is a connection between the solidification structure of remelted material and its plastic yield behaviour. This can be accounted for in the numerical forming simulation by the application of Barlat's yield criterion and the associated flow rule. This anisotropic material law was expanded to the use under hot working conditions, providing the possibility to additionally model an orientation dependency of the flow stress. Since anisotropic yield criteria generally have their origin in sheet metal forming analysis, the required material coefficients cannot easily be determined for the application to bulk metal forming. However, this rather phenomenological approach allows extensive parameter studies concerning the numerical interpretation of the underlying flow stress data and thus proved applicable to the upsetting process of cylindrical specimen.

\section{References}

[1] G. Wasle; COGGING, PhD Thesis, TU Graz; 2003

[2] M. Wolske, R. Kopp; Herstellungs- und Lebensdauermodelle für den Einsatz von Ni-BasisWerkstoffen in Dampfturbinen oberhalb von $700^{\circ} \mathrm{C}$, Work Report, RWTH Aachen; 1999

[3] Innovative Research Inc.; MeltFlow-VAR ${ }^{\mathrm{TM}}$ - Theoretical Basis and Use

[4] F. Barlat, D.J. Lege, J.C. Brem; A six-component yield function for anisotropic metals, International Journal of Plasticity, Vol. 7, pp. 693-712; 1991

[5] MSC.Software Corporation; Marc ${ }^{\circledR} 2007$ rl - Volume A: Theory and User Information 